\title{
Asymptotic SINR for Millimeter Wave Massive MIMO Cellular Networks
}

\author{
Tianyang Bai and Robert W. Heath, Jr. \\ Wireless Networking and Communication Group \\ The University of Texas at Austin \\ 1616 Guadalupe Street, C0803, Austin, TX 78701 \\ \{tybai, rheath\}@utexas.edu
}

\begin{abstract}
Thanks to the small wavelength at millimeter wave (mmWave) frequency, it is promising to combine massive multiple-input and multiple-output (MIMO) with mmWave. MmWave massive MIMO will differ from the conventional massive MIMO, due to the differences in propagation and hardware constraints. This paper proposes a stochastic geometry framework for evaluating the performance in large-scale mmWave massive MIMO networks. Based on the system model, analytical expressions are provided for the asymptotic signal-tointerference-plus-noise ratio (SINR) distributions in both uplink and downlink, when the number of base station antennas goes to infinity. Numerical results indicate a fast convergence in the SINR distribution to its asymptotic equivalence in dense mmWave networks. A comparison with conventional massive MIMO shows that mmWave massive MIMO achieves a higher cell throughput with sufficiently dense deployments.
\end{abstract}

\section{INTRODUCTION}

Massive multiple-input and multiple-output (MIMO) is a promising candidate technology for $5 \mathrm{G}$ cellular networks [1][3]. It deploys more antennas than in conventional systems to serve a large number of users and provides high throughput [1]-[4]. With large bandwidth channels, the millimeter wave (mmWave) spectrum constitutes a promising candidate frequency for access channels in 5G cellular networks [5], [6]. The small wavelength also makes it natural to consider massive MIMO at mmWave frequencies [7], which we call mmWave massive MIMO in this paper. MmWave cellular networks will be different from the system at lower frequencies. One key difference is the sensitivity to blockages: different path loss laws are found in the line-of-sight (LOS) and non-line-ofsight (NLOS) mmWave links in measurements [6]. Besides the differences in propagation, mmWave base stations will probably have fewer RF chains than conventional systems, due to power constraints. Consequently, a mmWave base station will support fewer users, and probably use analog or hybrid beamforming [8].

Stochastic geometry provides useful tools to analyze the system-level performance in large-scale networks [9]. In [10], [11], the asymptotic performance in a conventional massive MIMO network was examined using stochastic geometry, where key features of mmWave systems, e.g. the blockages and directional beamforming, were not incorporated. Stochastic geometric cellular models [9] were also extended to analyze mmWave network performance [12], [13]. An innovation of the analysis in [12], [13] was to incorporate building blockage into the analytical framework by using different path-loss laws for LOS and NLOS links. Unfortunately, the framework in [12], [13] cannot be directly applied to study mmWave massive MIMO networks, as intra-cell interference was not treated by assuming a single user per cell, and the channel training stage was not included by assuming perfect channel knowledge.

In this paper, we propose to study the asymptotic signalto-noise-and-interference ratio (SINR) and rate performance in a time-division duplex (TDD) mmWave massive MIMO system. We extend the mmWave cellular model in [12], [13] to the mmWave massive MIMO case by characterizing the distributions of the multiple scheduled users per cell and incorporating the difference in spatial correlations between LOS and NLOS links. Based on the system model, we derive expressions to evaluate the asymptotic SINR distributions in both uplink and downlink, when the number of base station antennas goes to infinity. Compared with prior asymptotic analysis of massive MIMO in [4], we incorporate key features in mmWave networks, including blockage effects and directional beamforming, and consider a different large-scale network topology with infinite randomly distributed base stations. Numerical results show that the SINR performance in massive MIMO networks is dependent on the base station density, where a good SINR coverage and high convergence rate to the asymptotic equivalence are achieved with a dense base station deployment. Moreover, a comparison with massive MIMO system at $2 \mathrm{GHz}$ shows that mmWave massive MIMO achieves a higher cell throughput when densely deployed.

\section{SySTEM MODEL}

In this section, we introduce the system model for a mmWave massive MIMO network. We consider a mmWave massive MIMO cellular network with perfect synchronization. Each base station is assumed to have $M$ antennas. In each time-frequency resource block, a base station can schedule $K$ users simultaneously in its cell. Let $X_{\ell}$ be the location of the $\ell$-th base station, $Y_{\ell}^{(k)}$ be the location of the $k$-th scheduled user in the cell of $\ell$-th base station, and $\mathbf{h}_{\ell \ell^{\prime}}^{(k)}$ the channel vector from $X_{\ell}$ to $Y_{\ell^{\prime}}^{(k)}$.

The network is assumed to be operated in the following TDD mode as proposed in [4]: channel training is performed in the uplink, where the users send their assigned pilots $\mathbf{T}_{k}$, and 
base stations estimate the channels using the orthogonality of the pilots; based on the channel estimates derived from uplink pilots, the base stations apply maximum ratio combining to receive the uplink data, and match-filter beamforming to transmit the downlink data. Further, we assume full reuse of the orthogonal pilots $\left\{\mathbf{T}_{k}\right\}_{1 \leq k \leq K}$ in the network. Due to the power and hardware constraints [8], mmWave base stations will have fewer RF chains than the antennas. To address the limitation on the number of RF chains, we restrict the number of simultaneously scheduled users in a cell to be smaller than 4 . Though mmWave systems will probably apply analog or hybrid beamforming, for simplicity, we assume digital beamforming and combining in this paper.

Now, we introduce the mmWave channel assumptions. One key feature of mmWave channels is the sensitivity to blockages: the presence of building blockages in urban areas makes the large-scale path loss laws much different in the LOS and NLOS links [6]. To distinguish the LOS and NLOS links, let $p_{\mathrm{L}}(R)$ be the probability that a link of length $R$ is LOS, and $p_{\mathrm{N}}(R)$ the probability that the link is NLOS. The LOS probability function $p_{\mathrm{L}}(R)$ is assumed to have finite first moment, i.e., $\int_{0}^{\infty} p_{\mathrm{L}}(r) r \mathrm{~d} r<\infty$, which is satisfied by most LOS probability functions in literatures, e.g. the ones in [14], [15]. The path loss $L(R)$ for a link of length $R$ is

$$
\begin{aligned}
& L(R)=\mathbf{1}\left[\psi<p_{\mathrm{L}}(R)\right] C_{\mathrm{L}}(\max (\delta, R))^{-\alpha_{\mathrm{L}}} \\
& +\mathbf{1}\left[p_{\mathrm{L}}(R) \leq \psi<\left(p_{\mathrm{L}}(R)+p_{\mathrm{N}}(R)\right)\right] C_{\mathrm{N}}(\max (\delta, R))^{-\alpha_{\mathrm{N}}},
\end{aligned}
$$

where $\mathbf{1}[\cdot]$ is the indicator function, $\psi$ is a uniform random variable in $[0,1], \delta=1$ meter is the reference distance, $\alpha_{\mathrm{L}}, \alpha_{\mathrm{N}}$ are the LOS and NLOS path loss exponents, and $C_{\mathrm{L}}, C_{\mathrm{N}}$ are the intercepts in the LOS and NLOS path loss. Typical values of mmWave path loss parameters are available in measurement papers [6], [16]. In addition, we assume for different links, the random variable $\psi$, which decides whether the link is LOS or not, are independent.

For small-scale fading, we consider narrowband channels, as the effect of frequency selective fading can be minimized by techniques like orthogonal frequency-division multiplexing (OFDM) or frequency domain equalization [17]. Measurements show that small-scale fading has minor effects on LOS mmWave signals [6]. Consequently, for LOS links, we model the channel vector $\mathbf{h}_{\ell \ell^{\prime}}^{(k)}$ by a deterministic vector as

$$
\mathbf{h}_{\ell \ell^{\prime}}^{(k)}=\sqrt{M} \beta_{\ell \ell^{\prime}}^{(k) 1 / 2} \mathbf{u}_{\ell \ell^{\prime}}^{(k)},
$$

where $\beta_{\ell \ell^{\prime}}^{(k)}$ is the path loss computed from (1), assuming the link is LOS, i.e., $\psi<p_{\mathrm{L}}(R)$; $\mathbf{u}_{\ell \ell^{\prime}}^{(k)}$ is a unit vector. Furthermore, we assume asymptotic orthogonality between any two LOS channels as for $(s, t, k) \neq\left(s^{\prime}, t^{\prime}, k^{\prime}\right)$,

$$
\lim _{M \rightarrow \infty} \mathbf{u}_{s t}^{(k) *} \mathbf{u}_{s^{\prime} t^{\prime}}^{\left(k^{\prime}\right)}=0 .
$$

One example satisfying (3) is the LOS channels using uniform linear arrays with non-overlapping angles of arrival [18].

For the NLOS channel, we apply a correlated fading model to account for the potentially larger number of scatters. We express the NLOS channel vector as

$$
\mathbf{h}_{\ell n}^{(k)}=\left(\beta_{\ell n}^{(k)}\right)^{1 / 2} \boldsymbol{\Phi}_{\ell n}^{(k) 1 / 2} \mathbf{w}_{\ell n}^{(k)},
$$

where $\beta_{\ell n}^{(k)}$ is the large-scale path loss, $\mathbf{w}_{\ell n}^{(k)}$ is the fading vector consisting of identically and independently distributed (IID) random variables with zero mean and unit variance, and $\boldsymbol{\Phi}_{\ell n}^{(k)}$ is the covariance matrix to account for correlations in small-scale fading. Let $\lambda_{\ell n}^{(k)}[m]$ be the eigenvalues of the covariance matrix $\boldsymbol{\Phi}_{\ell n}^{(k)}$. We assume the traces of the covariance matrices for all channels are normalized to $M$, i.e., Trace $\left[\boldsymbol{\Phi}_{\ell n}^{(k)}\right]=\sum_{m=1}^{M} \lambda_{\ell n}^{(k)}[m]=M$, and the average squares of the eigenvalues are upper bounded by a constant $\gamma$ : $\frac{1}{M} \sum_{m=1}^{M} \lambda_{\ell n}^{(k) 2}[m] \leq \gamma$, which is satisfied by many common channel models, including the case of uniform linear arrays with half-wavelength spacing and continuous angular spread [19].

Next, we describe the stochastic geometric network model. We assume the base stations are distributed as a Poisson point process (PPP) with density $\lambda_{\mathrm{b}}$. The users, either scheduled or not, are distributed as an independent PPP on the plane with sufficiently high density, such that each base station is associated with at least $K$ users. A user is assumed to be associated with the base station that provides the minimum path loss signal. Without loss of generality, a typical scheduled user $Y_{0}^{(1)}$ is fixed at the origin. We will investigate the SINR and rate performance at this typical user.

Now we focus on the distribution of scheduled users. In a resource block, let $\mathcal{N}_{\mathrm{u}}^{(k)}$ be the point process formed by the locations of the scheduled users $Y_{\ell}^{(k)}$, i.e., all the scheduled users assigned with the $k$-th pilot sequence. Note that though the users are distributed as a PPP on the plane, the scheduled users do not form a PPP, as their locations are correlated. For instance, in each cell, the number of scheduled users is fixed to be $K$, while for a PPP, the number of points in the same region is randomly distributed. The correlations in the scheduled users' location make the exact analysis intractable. Therefore, we make the following approximation on the distribution of $\mathcal{N}_{\mathrm{u}}^{(k)}$.

Approximation 1: The path losses $\beta_{\ell \ell}^{(k)}$ from the users to their associated base stations are assumed to be IID. Moreover, the tagged base station $X_{0}$ is assumed to observe the othercell scheduled users in $\mathcal{N}_{\mathrm{u}}^{(k)}$ as a thinned Poisson point process with intensity function

$$
\lambda_{\mathrm{u}}\left(\left|X_{0}-x\right|\right)=\lambda_{\mathrm{b}} \mathbf{1}\left(L\left(\left|X_{0}-x\right|\right)>\beta_{x}\right),
$$

where $L\left(\left|X_{0}-x\right|\right)$ represents the path loss from $x$ to $X_{0}, \beta_{x}$ is an IID random variable with the same distribution as $\beta_{00}^{(1)}$, and the indicator function $\mathbf{1}\left(L\left(\left|X_{0}-x\right|\right)>\beta_{x}\right)$ ensures that any user outside the tagged cell $X_{0}$ has smaller path loss to its own base station than to $X_{0}$. Besides, the scheduled users assigned with different pilots are assumed to be independently distributed, i.e., for $k \neq k^{\prime}, N_{\mathrm{u}}^{(k)}$ and $N_{\mathrm{u}}^{\left(k^{\prime}\right)}$ are independent.

It can be shown in simulations that the proposed approximation provides a close characterization of the actual scheduled 
users in a network with PPP distributed base stations [20].

MmWave handsets will use antenna arrays to perform directional beamforming [5]. To simplify the analysis, the antenna array at the mobile station is modeled as a single directional antenna with a gain pattern approximated by the sectored antenna pattern. In the sectored antenna model, the directivity gain within the main lobe $\theta$ is assumed to be a constant $Q$, while all angles outside the main lobe $\theta$ have the constant side lobe gain $q$. Let $D_{\ell \ell^{\prime}}^{(k)}$ be the directivity gain of the mobile station $Y_{\ell^{\prime}}^{(k)}$ to base station $X_{\ell}$. We assume the directions of the mobile station antennas are adjusted to maximize the desired signal power without alignment errors. Moreover, for the interfering links, we assume the antenna directions are independently and uniformly distributed. Consequently, the directivity gain $D_{\ell \ell}^{(k)}=Q$ in the desired link; for other interfering links, $D_{\ell \ell^{\prime}}^{(k)}$ is a Bernoulli random variable, where $D_{\ell \ell^{\prime}}^{(k)}=Q$ with probability $\frac{\theta}{2 \pi}$, and $D_{\ell \ell^{\prime}}^{(k)}=q$ otherwise.

Last, we assume that the base stations estimate the channels by correlating the received signals with the pilots, and do not use MMSE estimation. Hence, the channel estimate of $\mathbf{h}_{\ell \ell}^{(k)}$ at base station $X_{\ell}$ is

$$
\overline{\mathbf{h}}_{\ell \ell}^{(k)}=\sqrt{Q} \mathbf{h}_{\ell \ell}^{(k)}+\sum_{\ell^{\prime} \neq \ell} D_{\ell \ell^{\prime}}^{(k) 1 / 2} \mathbf{h}_{\ell \ell^{\prime}}^{(k)}+\mathbf{n}_{\mathrm{T}},
$$

where $\mathbf{n}_{T}$ is a Gaussian noise vector of the distribution $\mathcal{C N}\left(\mathbf{0}, \frac{1}{K \rho_{\mathrm{T}}} \mathbf{I}\right)$, and $\rho_{\mathrm{T}}$ is the signal-to-noise ratio $(\mathrm{SNR})$ in the channel training.

Based on the proposed system model, we will analyze the SINR and rate performance in both uplink and downlink in the subsequent sections.

\section{SINR COVERAGE ANALYSIS}

In this section, we derive analytical expressions to approximate the distributions of the asymptotic SINR, when the number of base station antennas goes to infinity.

\section{A. Uplink Analysis}

In this section, we analyze the uplink SINR performance in mmWave massive MIMO networks. First, we compute the density function of scheduled users in (5) explicitly as follows.

Lemma 1: The density function $\lambda_{\mathrm{u}}(r)$ of the other-cell scheduled users in $\mathcal{N}_{\mathrm{u}}^{(k)}$ can be computed as

$$
\lambda_{\mathrm{u}}(r)=\lambda_{\mathrm{u}, \mathrm{L}}(r)+\lambda_{\mathrm{u}, \mathrm{N}}(r),
$$

where for $\mathrm{s} \in\{\mathrm{L}, \mathrm{N}\}, \lambda_{\mathrm{u}, \mathrm{s}}(r)=A \lambda_{\mathrm{b}} p_{\mathrm{s}}(r)\left(1-\mathrm{e}^{-\Xi\left(r^{\alpha_{\mathrm{s}}} / C_{\mathrm{s}}\right)}\right)$, $A=1-\mathrm{e}^{-2 \pi \lambda_{\mathrm{b}} \int_{0}^{\infty}\left(p_{\mathrm{L}}(x)+p_{\mathrm{N}}(x)\right) x \mathrm{~d} x}$, and

$$
\Xi(t)=2 \pi \lambda_{\mathrm{b}}\left(\int_{0}^{\left(t C_{\mathrm{N}}\right)^{1 / \alpha_{N}}} r p_{\mathrm{N}}(r) \mathrm{d} r+\int_{0}^{\left(t C_{\mathrm{L}}\right)^{1 / \alpha_{L}}} r p_{\mathrm{L}}(r) \mathrm{d} r\right) .
$$

Proof: See [20].

To decode the uplink symbol $s_{0}^{(1)}$ sent by $Y_{0}^{(1)}$, the base station $X_{0}$ applies maximum ratio combining, based on the channel $\overline{\mathbf{h}}_{00}^{(1)}$. Then, the uplink SINR for the user $Y_{0}^{(1)}$ is

$\frac{\left|\overline{\mathbf{h}}_{00}^{(1) *} \mathbf{h}_{00}^{(1)}\right|^{2}}{\sum_{\ell \neq 0}\left|\overline{\mathbf{h}}_{00}^{(1) *} \mathbf{h}_{0 \ell}^{(1)}\right|^{2}+\sum_{k=2}^{K} \sum_{\ell>0}\left|\overline{\mathbf{h}}_{00}^{(1) *} \mathbf{h}_{0 \ell}^{(k)}\right|^{2}+\left|\overline{\mathbf{h}}_{00}^{(1) *} \mathbf{h}_{\mathrm{u}}\right|^{2}}$, where $\mathbf{n}_{U}$ is the noise vector of the distribution $\mathcal{C N}\left(\mathbf{0}, \rho_{\mathrm{U}}^{-1} \mathbf{I}\right)$, and $\rho_{\mathrm{U}}$ is the SNR in uplink data transmission. When the number of antennas $M$ goes to infinity, the uplink SINR converges to its asymptotic equivalence as follows.

Theorem 1: When $M \rightarrow \infty$, the uplink SINR converges in probability to

$$
\operatorname{SINR}_{\mathrm{UL}} \stackrel{p}{\rightarrow} \frac{Q^{2} \beta_{00}^{(1) 2}}{\sum_{\ell \neq 0} D_{0 \ell}^{(1) 2} \beta_{0 \ell}^{(1) 2}}
$$

Proof: See [20].

The result in Theorem 1 is different from prior work in [4] in that (i) we consider a large-scale network with infinite randomly located base stations, in which case the analysis based on a network topology with finite base stations in [4] does not directly apply; (ii) we consider different spatial correlations in fading for the LOS and NLOS channels, while IID fading was assumed in [4]. Consequently, mathematical tools from stochastic geometry, including the factorial moment and Campbell's formula, are required to prove Theorem 1 [20]. The same comment also applies to Theorem 2 in the downlink analysis.

Next, we derive a tight approximation for the asymptotic SINR distribution in the following corollary.

Corollary 1.1: The distribution of the asymptotic SINR in (6) can be approximated as

$$
\begin{aligned}
\mathbb{P}\left(\operatorname{SINR}_{\mathrm{UL}}>T\right) & \approx A \sum_{n=1}^{N}\left(\begin{array}{l}
N \\
n
\end{array}\right)(-1)^{n+1} \times \\
& \int_{0}^{\infty} \mathrm{e}^{-W_{n}(T, t)-V_{n}(T, t)-\Xi(t)} \Xi(\mathrm{d} t),
\end{aligned}
$$

where

$$
\begin{aligned}
W_{k}(T, t) & =\sum_{\ell=1}^{2} 2 \pi B_{\ell} \int_{0}^{\infty}\left(1-\mathrm{e}^{-k \eta T C_{\mathrm{L}} \xi_{\ell} t^{2} x^{-2 \alpha_{L}}}\right) \lambda_{\mathrm{u}, \mathrm{L}}(x) x \mathrm{~d} x, \\
V_{k}(T, t) & =\sum_{\ell=1}^{2} 2 \pi B_{\ell} \int_{0}^{\infty}\left(1-\mathrm{e}^{-k \eta T C_{\mathrm{N}} \xi_{\ell} t^{2} x^{-2 \alpha_{N}}}\right) \lambda_{\mathrm{u}, \mathrm{N}}(x) x \mathrm{~d} x,
\end{aligned}
$$

$A$ is as defined in Lemma $1, N$ is the number of truncated terms used in the approximation, $\eta=N(N !)^{-\frac{1}{N}}$; for $\ell=1,2$, $B_{\ell}=\left\{\frac{\theta}{2 \pi}, 1-\frac{\theta}{2 \pi}\right\}$, and $\xi_{\ell}=\left\{1, q^{2} / Q^{2}\right\}$.

Note that the expression in Corollary 1.1 can be further simplified when provided the explicit forms of $p_{\mathrm{L}}(r)$ and $p_{\mathrm{N}}(r)$, e.g. the step functions in [12, Section III-D]. Moreover, the expression becomes more accurate when more truncated terms (a larger $N$ ) is used; simulations indicate that using $N \geq 5$ terms is sufficient to provides a tight approximation.

\section{B. Downlink SINR Analysis}

In this section, we investigate the downlink asymptotic SINR in mmWave massive MIMO networks. Let $\mathbf{f}_{\ell}^{(k)}$ be the beamforming precoder that base station $X_{\ell}$ applies for its user $Y_{\ell}^{(k)}$. When applying match-filter beamforming, it follows that $\mathbf{f}_{\ell}^{(k)}=\frac{\overline{\mathbf{h}}_{\ell \ell}^{(k)}}{\left\|\overline{\mathbf{h}}_{\ell \ell}^{(k)}\right\|}$. Then the downlink SINR at the typical user 


$$
Y_{0}^{(1)} \text { is } \frac{\left|\mathbf{h}_{00}^{(1) *} \mathbf{f}_{0}^{(1)}\right|^{2}}{\sum_{\ell \neq 0}\left|\mathbf{h}_{\ell 0}^{(1) *} \mathbf{f}_{\ell}^{(1)}\right|^{2}+\sum_{k \neq 1}^{K} \sum_{\ell \geq 0}\left|\mathbf{h}_{\ell 0}^{(1) *} \mathbf{f}_{\ell}^{(k)}\right|^{2}+\rho_{\mathrm{D}}^{-1}},
$$

where $\rho_{\mathrm{D}}$ is the SNR in the downlink transmission. We have the following convergence result on the downlink SINR.

Theorem 2: With match-filter beamforming, the downlink SINR will converge in probability to its asymptotic equivalence as $\lim _{M \rightarrow \infty} \operatorname{SINR}_{\mathrm{DL}} \stackrel{p}{\rightarrow} \frac{Q^{2} \beta_{00}^{(1) 2} / a_{0}^{(1)}}{\sum_{\ell \neq 0} D_{\ell 0}^{(1) 2} \beta_{\ell 0}^{(1) 2} / a_{\ell}^{(1)}}$, where $a_{\ell}^{(k)}=\frac{1}{K \rho_{\mathrm{T}}}+\sum_{\ell^{\prime}} D_{\ell \ell^{\prime}}^{(k)} \beta_{\ell \ell^{\prime}}^{(k)}$.

The exact distribution of the asymptotic SINR in Theorem 2 is generally difficult to derive, as the normalization constants $a_{\ell}^{(k)}$ introduce correlations among all the terms. In a dense mmWave network, however, the asymptotic SINR can be approximated by the following corollary.

Corollary 2.1: In a dense mmWave network, the asymptotic downlink SINR distribution can be approximated as

$$
\mathbb{P}(\mathrm{SINR}>T) \approx A \sum_{n=1}^{N}\left(\begin{array}{l}
N \\
n
\end{array}\right)(-1)^{n+1} \int_{0}^{\infty} \mathrm{e}^{-Z_{n}(T, t)-\Xi(t)} \Xi(\mathrm{d} t),
$$

where $Z_{k}(T, t)=\sum_{\ell=1}^{2} B_{\ell} \int_{t}^{\infty}\left(1-\mathrm{e}^{-k \eta T \xi_{\ell} t^{2} x^{-2}}\right) \Xi(\mathrm{d} x)$, $A, \eta, B_{\ell}$, and $\xi_{\ell}$ are the same as defined in Corollary 1.1, and $N$ is the number of terms used in the approximation.

Last, we define the average achievable rate at a typical user as $\Gamma=W(1-\mu) \log _{2}\left(1+\min \left\{\operatorname{SINR}, T_{\max }\right\}\right)$, where $W$ is the bandwidth assigned to a user, $\mu$ is the fraction of overhead, and $T_{\max }$ is a SINR distortion threshold. In an OFDM massive MIMO system, the fraction of overhead $\mu$ can be computed based on [4, Section III-A], which accounts for the inefficiency from cyclic prefix and uplink training. The use of a distortion threshold $T_{\max }$ is needed because of the potential for very high SINRs in massive MIMO networks that may not be exploited due to other limiting factors like linearity in the radio frequency front-end. By [9], given the SINR distribution $\mathbb{P}(\mathrm{SINR}>T)$, the average achievable rate can be computed as $\mathbb{E}[\Gamma]=\frac{W}{\ln 2} \int_{0}^{T_{\max }} \frac{\mathbb{P}(\text { SINR }>t)}{1+t} \mathrm{~d} t$.

\section{NumERicAl RESUlts}

In this section, we consider a $28 \mathrm{GHz}$ massive MIMO network with a system bandwidth of $500 \mathrm{MHz}$. We assume the transmitting power is $30 \mathrm{dBm}$ in the downlink, and $20 \mathrm{dBm}$ in the uplink. In the Monte Carlo simulations, the density of the user process is assumed to be 60 times the base station density, and a mmWave base station randomly serves $K=4$ users in its cell in a resource block. Based on the New York city measurement in [16], we assume $p_{\mathrm{L}}(r)=\mathrm{e}^{-\xi r} \mathbf{1}\left(r<R_{\mathrm{O}}\right)$ and $p_{\mathrm{N}}(r)=\left(1-\mathrm{e}^{-\xi r}\right) \mathbf{1}\left(r<R_{\mathrm{O}}\right)$, where $\xi=70$ meters, and $R_{\mathrm{O}}=200$ meters. In addition, we assume the LOS path loss exponent is $\alpha_{\mathrm{L}}=2$, and the NLOS path loss exponent is $\alpha_{\mathrm{N}}=4$. For the channel models, we use the steering vectors of uniform linear array with half-wavelength spacing as LOS channel vectors; for NLOS channels, we assume IID Rayleigh fading for simplicity.

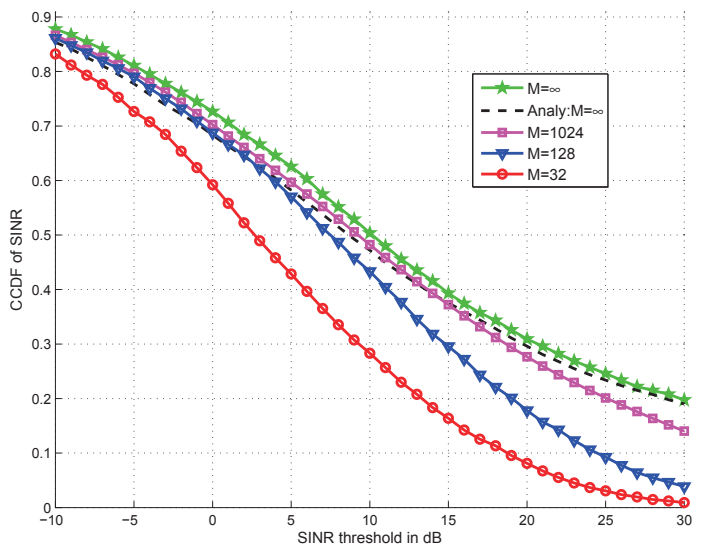

Fig. 1. Uplink SINR distributions in dense mmWave networks. In the simulations, we assume ISD $=100$ meters. The analytical curve is drawn based on Corollary 1.1.

First, we show the convergence of uplink SINR in a dense mmWave network in Fig. 1. In the simulations, we assume each base station schedule $K=4$ users, and mobile stations use omni-directional antennas. As shown in Fig. 1(a), in a dense mmWave network with ISD $=100$ meters, the asymptotic SINR distribution provides a close characterization of the SINR curve with $M=1024$ antennas. Besides, the simulation shows that the analytical expression in Corollary 1.1 provides a tight approximation.

Next, we present the numerical results for the downlink SINR. In Fig. 2, a comparison between the performance in dense and sparse mmWave networks shows that due to the presence of blockages and high noise power, the SINR performance is much sensitive to the base station density, and mmWave massive MIMO networks require dense base station deployments to achieve good coverage. Moreover, the downlink SINR in dense mmWave networks is shown to converge much faster than that in the sparse network. In addition, in Fig. 2 (a), we show that Corollary 2.1 provides a tight approximation of the asymptotic SINR distribution in dense mmWave networks.

Last, we compare the average rate of massive MIMO systems at $2 \mathrm{GHz}$ and $28 \mathrm{GHz}$ in Table I. In the rate comparison, we assume the $2 \mathrm{GHz}$ base stations have $M=64$ antennas, and $W=100 \mathrm{MHz}$ bandwidth, while in mmWave systems $M=128$, and $W=500 \mathrm{MHz}$. Both the uplink and downlink are assumed to take up $50 \%$ transmission time. The OFDM technique is assumed to be used in both systems: the parameters for the $2 \mathrm{GHz}$ system are taken from the LTE standard [15], and those for mmWave systems are from [21]. Besides, the training overhead is computed based on [4]. Further details for the rate comparison can be found in [20]. The results show that though serving fewer users per cell, mmWave massive MIMO still outperforms conventional massive MIMO systems in cell throughput, due to the larger bandwidth. 


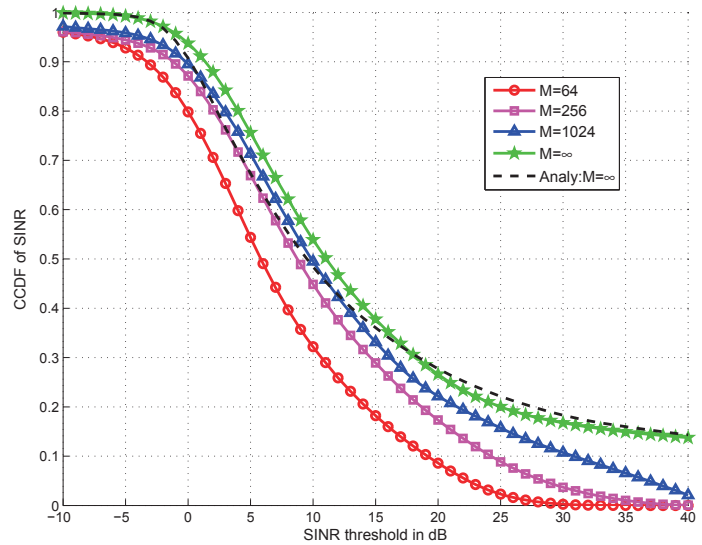

(a) Downlink SINR in dense networks.

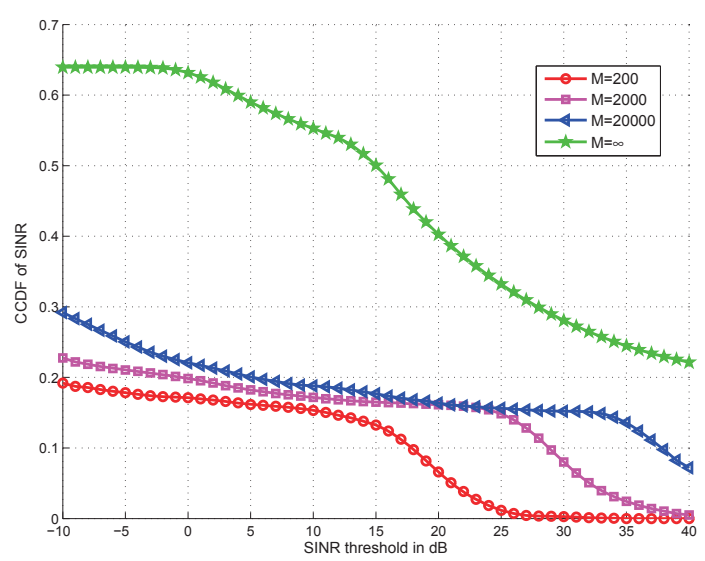

(b) Downlink SINR in sparse networks.

Fig. 2. Downlink mmWave SINR distributions with different base station densities. We assume ISD $=100 \mathrm{~m}$ in (a), and ISD $=400 \mathrm{~m}$ in (b). The analytical curve in (a) is drawn based on Corollary 2.1.

TABLE I

Comparison of ACHIEVABle Rates

\begin{tabular}{c|c|c|c}
\hline Carrier & $2 \mathrm{GHz}$ & $28 \mathrm{GHz}$ & $28 \mathrm{GHz}$ \\
\hline Avg. ISD (m) & 500 & 100 & 400 \\
\hline Training overhead & $20 \%$ & $14 \%$ & $14 \%$ \\
\hline Bandwidth (MHz) & 100 & 500 & 500 \\
\hline Rate per user (Mbps) & 52.8 & 1791.0 & 436.5 \\
\hline Users per cell & 14 & 4 & 4 \\
\hline Cell throughput (Mbps) & 740.0 & 7164.0 & 1745.8 \\
\hline
\end{tabular}

\section{CONCLUSiOnS}

In this paper, we analyzed the asymptotic SINR distribution in mmWave massive MIMO networks by incorporated key features of mmWave systems, including the blockage effects and directional beamforming at mobile stations, into the analytical framework. We provided the asymptotic equivalences for both the uplink and downlink SINR in a large-scale network with Poisson distributed base stations, and derived approximation expressions to compute their distributions. The accuracy of the analytical expressions were verified by numerical simulations. The numerical results showed that mmWave massive MIMO requires a high base station density to achieve good SINR coverage. Moreover, the comparison with massive MIMO systems at lower frequencies showed the promising gain of mmWave massive MIMO over conventional massive MIMO in cell throughput. For future work, it would be interesting to incorporate mmWave hardware constraints, such as hybrid beamforming and one-bit A/D converter [8].

\section{ACKNOWLEDGEMENT}

This paper is based upon work supported by the National Science Foundation under Grants No. 1218338 and 1319556.

\section{REFERENCES}

[1] E. Larsson, O. Edfors, F. Tufvesson, and T. Marzetta, "Massive MIMO for next generation wireless systems," IEEE Communications Magazine, vol. 52, no. 2, pp. 186-195, Feb. 2014.

[2] F. Boccardi, R. Heath, A. Lozano, T. Marzetta, and P. Popovski, "Five disruptive technology directions for 5G," IEEE Communications Magazine, vol. 52, no. 2, pp. 74-80, February 2014.

[3] L. Lu, G. Li, A. Swindlehurst, A. Ashikhmin, and R. Zhang, "An overview of massive MIMO: Benefits and challenges," IEEE J. Sel. Topics Signal Process., vol. 8, no. 5, pp. 742-758, Oct. 2014.

[4] T. Marzetta, "Noncooperative cellular wireless with unlimited numbers of base station antennas," IEEE Trans. Wireless Commun., vol. 9, no. 11, pp. 3590-3600, Nov. 2010.

[5] Z. Pi and F. Khan, "An introduction to millimeter-wave mobile broadband systems," IEEE Communications Magazine, vol. 49, no. 6, pp. 101-107, June 2011.

[6] T. Rappaport et al., "Millimeter wave mobile communications for $5 \mathrm{G}$ cellular: It will work!" IEEE Access, vol. 1, pp. 335-349, 2013.

[7] A. Swindlehurst, E. Ayanoglu, P. Heydari, and F. Capolino, "Millimeterwave massive MIMO: the next wireless revolution?" Communications Magazine, IEEE, vol. 52, no. 9, pp. 56-62, September 2014.

[8] A. Alkhateeb, J. Mo, N. Gonzalez-Prelcic, and R. Heath, "MIMO precoding and combining solutions for millimeter-wave systems," IEEE Communications Magazine, vol. 52, no. 12, pp. 122-131, Dec. 2014.

[9] J. Andrews et al., "A tractable approach to coverage and rate in cellular networks," IEEE Trans. Commun., vol. 59, no. 11, pp. 3122-3134, 2011.

[10] P. Madhusudhanan, X. Li, Y. Liu, and T. Brown, "Stochastic geometric modeling and interference analysis for massive MIMO systems," in Proc.of Modeling Optimization in Mobile, Ad Hoc Wireless Networks (WiOpt), May 2013, pp. 15-22.

[11] T. Bai and R. W. Heath Jr., "Asymptotic coverage probability and rate in massive MIMO networks," in Proc. of IEEE Global Conf. on Signal and Information Processing (GlobalSIP), Dec. 2014.

[12] T. Bai and R. Heath Jr., "Coverage and rate analysis for millimeter-wave cellular networks," IEEE Trans. Wireless Commun., vol. 14, no. 2, pp. 1100-1114, Feb. 2015.

[13] T. Bai, A. Alkhateeb, and R. W. Heath Jr, "Coverage and capacity in millimeter wave cellular networks," IEEE Commun. Mag., Sep. 2014.

[14] T. Bai, R. Vaze, and R. W. Heath Jr., "Analysis of blockage effects on urban cellular networks," IEEE Trans.Wireless Commun., vol. 13, no. 9, pp. 5070-5083, Sep. 2014

[15] 3GPP TR 36.814, "Further advancements for E-UTRA physical layer aspects (Release 9)," Mar. 2010.

[16] M. Akdeniz, Y. Liu, M. Samimi, S. Sun, S. Rangan, T. Rappaport, and E. Erkip, "Millimeter wave channel modeling and cellular capacity evaluation," IEEE J. Sel. Areas Commun., vol. 32, no. 6, pp. 1164-1179, June 2014.

[17] A. Goldsmith, Wireless Communications. Cambridge University Press, 2005.

[18] H. Q. Ngo, E. Larsson, and T. Marzetta, "Aspects of favorable propagation in massive MIMO," in Proc. of European Signal Processing Conf. (EUSIPCO), Sep. 2014, pp. 76-80.

[19] A. Adhikary, J. Nam, J.-Y. Ahn, and G. Caire, "Joint spatial division and multiplexing: The large-scale array regime," IEEE Trans. on Information Theory, vol. 59, no. 10, pp. 6441-6463, Oct. 2013.

[20] T. Bai and R. W. Heath Jr., "Massive MIMO: Millimeter wave or lower frequencies?" Preprint, Feb. 2015.

[21] Z. Pi and F. Khan, "A millimeter-wave massive MIMO system for next generation mobile broadband," in Proc. of the Forty Sixth Asilomar Conf. on Signals, Systems and Computers (ASILOMAR), 2012, pp. 693-698. 